\title{
Microwave imaging of skin damage at experimental burns
}

\author{
$\underline{\text { Andrew K. Martusevich }}^{1}$, A.G. Galka ${ }^{1,2}$, S.Yu. Krasnova ${ }^{1}$, A.G. Soloveva ${ }^{1}$ \\ ${ }^{1}$ Privolzhsky Research Medical University, Nizhny Novgorod, Russia, cryst-mart@yandex.ru \\ ${ }^{2}$ Institute of applied Physics, Nizhny Novgorod, Russia
}

The ubiquitous prevalence of thermal injury predetermines not only the search and testing of innovative technologies for the treatment of severely burned, but also the improvement of the diagnostic apparatus of combustiology $[1,2,4]$. Currently, the clinical assessment of the local status has the greatest importance for the considered contingent of victims, including the one associated with the use of a number of empirical algorithms to determine the spatial characteristics of the injury ("rule of nines", etc.) [1, 2]. On the other hand, in combustiology there is a significant number of diagnostic difficulties associated with the specification of the boundaries of burn injury, tissue viability in the near-wound zone, wound uniformity, etc. [1, 2, 4]. Verification of the depth of skin and subcutaneous structures lesions should be singled out as a separate item [1-4]. In order to solve this complex of problems, in addition to the empirical approach prevailing in real clinical practice, the possibilities of thermal IR-imaging were studied [1, 3, 6]. It is shown that this technology is informative in a number of situations, but it allows us to judge only the state of the skin surface and the nearest underlying structures. Modern variants of ultrasound examination, which have high informative value and resolving ability in other pathology, do not allow to achieve the necessary contrast [3] in relation to the thermal injury.

An additional complicating factor in tissue imaging in combustiology is the presence of a physical barrier between the sensor and the surface of the skin (temporary and permanent wound coatings), which is not possible to eliminate for diagnostic manipulations (for example, when using biopo-coatings containing matrix with stem cells).) [1-3]. This is an obstacle for most methods of investigation of subsurface structure and blood tissues, in particular, for ultrasound examination. Therefore, it is necessary to search and test fundamentally different technologies of the assessment of the deep characteristics of the burn wound and the near-wound zone [2-4].

In this aspect, the method of near-field resonance microwave profiling, which has recently appeared in biomedicine and is based on the study of the dielectrical properties of tissues (dielectric permeability and conductivity) is very interesting [5]. Previous studies have shown that this technology is high informative in dermatology, allowing how to carry out primary diagnostics and differential diagnostics of various skin diseases and to monitor the efficacy of treatment, predicting the occurrence of the patient in the remission phase [5]. At the same time in combustiology the considered method was not applied earlier.

The purpose of this study is to evaluate the diagnostic capabilities of near-field microwave sensing in the estimation of the deep structure of the skin in the norm and in experimental burn wounds.

\section{Material and methods}

The study was performed on 30 male rats of the Wistar line, divided into 2 equal groups. The first group of animals $(n=15)$ was a control group (no manipulations were performed, except for a single microwave sounding). Rats in the second group $(n=15)$ was simulated thermal contact burns on $20 \%$ body square percent with our methodology (Peretyagin S.P. et al., 2009) [6]. The dielectrical properties of burn wound tissues were studied immediately after the application of burn and on 1 day after it.

Near-field microwave sensing of tissues was performed using a special device created at the Institute of Applied Physics of the Russian Academy of Sciences (Nizhny Novgorod), as well as specialized software, matching the installation with a PC and allowing the calculation of the real part of the dielectic permeability [5]. Dielectric characteristics of the skin were assessed at depths of 2 to $5 \mathrm{~mm}$ using a series of probes. All animals were measured at one point, localized in the middle part of the back, on a preepilated surface.

The results were processed using the program Statistica 6.0.

\section{The results and discussion}

The conducted research allowed to establish that it is possible to carry out the analysis of dielectric properties of skin of rats by means of the studied hardware-software complex.

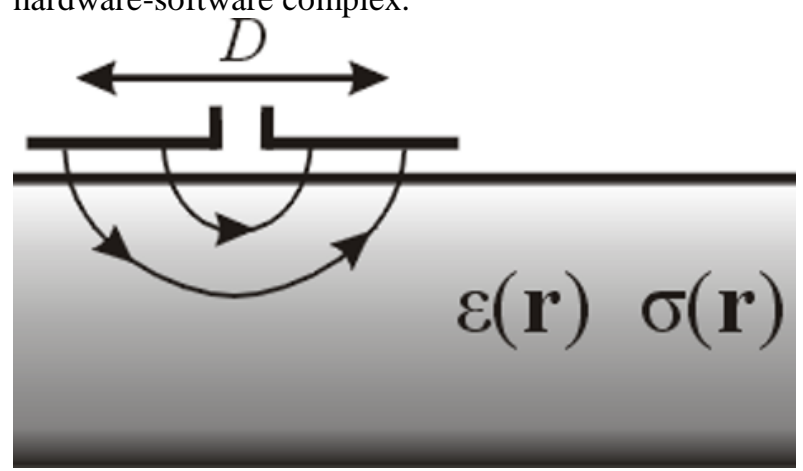

Fig. 1. Scheme of near-field resonanse microwavw sensing of subsurface structure (D - diameter of the sensor; $\varepsilon(\mathrm{r})-$ dielecric permittivity; $\sigma(\mathrm{r})$ - conductivity)

At the same time, it was possible to show that at the studied depths $(2-5 \mathrm{~mm})$ the level of dielectric conductivity of subsurface structures is at values less than 9 rel. un., monotonically increasing with increasing depth of sensing.

Given that each value is cumulative, i.e. includes the conductivity of the entire subsurface layer up to the specified depth (Fig. 1), the maximum level of the parameter is recorded at a distance of $5 \mathrm{~mm}$ from the 
surface of the skin. This is reflected in the character of the method of sounding as near-field (Fig. 1).

At the same time, the level of the studied parameter at the minimum and maximum depths differs by 2.55 times $(\mathrm{p}<0.01)$, which is associated with the assessment of deeper structures in rats during microwave profiling by one sensor performing sensing at a depth of $5 \mathrm{~mm}$. This is due to the fact that each subsequent value of the dielectric conductivity includes the previous one in conjunction with the contribution made by tissues located from the previous to the current level of sensing [11, 19-21].

Based on the data obtained from intact rats, a linear mathematical model of changes in the dielectric permittivity of animal skin is constructed, which sufficiently describes its subsurface profile (determination coefficient -0.91 ). The linear regression equation allows to predict the value of dielectric permittivity at other sensing depths. This model can be used to calculate the physiological level of dielectric conductivity of the subsurface structures of the skin of rats, used as a guide to identify its changes caused by various pathological processes.

The features of the deep structure of the dielectric properties of the skin and subcutaneous tissues in animals with modeled thermal injury (in the form of a thermal burn on the previously epilated surface of the back skin) were also studied. Evaluation of the dielectric permittivity of the subsurface tissues of the experimental burn wound was carried out by us immediately after the injury and on 1 day after its simulation. This allowed to form deep skin profiles by this parameter in the dynamics of the experiment and in comparison with intact biological tissue (Fig. 2).

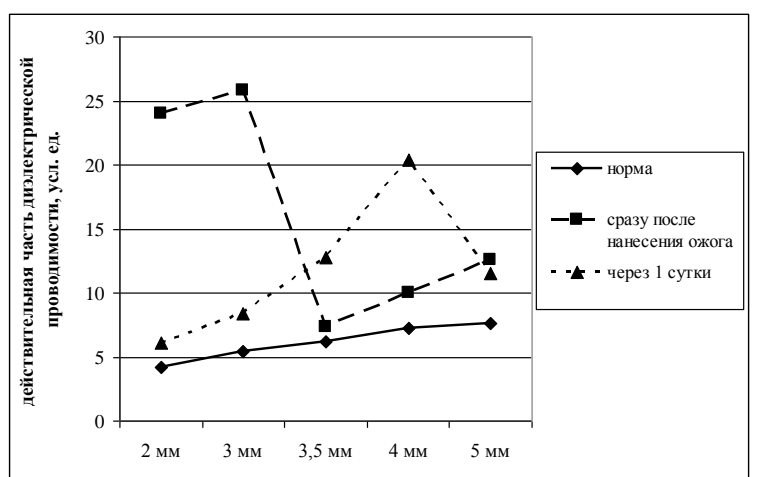

Fig. 2. The profile of dielectric permittivity of rats skin an subcutaneous tissues in normal conditions and after the burn

It was found that the dielectric properties of the burn wound differ significantly from the intact cover tissue. These shifts are characterized by an increase in the real part of the dielectric permittivity of the medium at both observation points. Thus, immediately after the application of thermal trauma, the greatest changes occur in the nearest subsurface layers of the skin $(2-3 \mathrm{~mm}$.), in which the figure under consideration repeatedly increases relative to intact rats $(7.46$ and 9.47 times at the depths of sensing 2 and $3 \mathrm{~mm}$; $\mathrm{p}<0.05$ for both cases), amounting to about 24.0 and
25.8 units, respectively. This may be due to the rapid intensive local heating of the tissues at a shallow depth immediately after exposure, whereas this effect has not yet spread to the deeper layers.

In 1 day after the burn the depth profile of the dielectric conductivity of the skin was significantly transformed (Fig. 3). During this period, there is a deepening of the lesion of subsurface structures, which is accompanied by a decrease in the level of the studied parameter at minimum depths $(2-3 \mathrm{~mm}$.) with its increase relative to the intact skin at a distance of $3.5-5 \mathrm{~mm}$ below the skin surface $(\mathrm{p}<0.05$ for all cases). At the same time, the maximum dielectric conductivity was recorded at a depth of $4 \mathrm{~mm}$, which in our experiments corresponded to the zone of greatest damage. This indicates a partial cooling of the surface layers of the skin with simultaneous overheating of more proximally lying and, consequently, a shift in the focus of damage to the deeper layers. The presented data indicate the possibility of monitoring the depth of thermal tissue damage, including creating an experimental basis for testing the processes of deepening burn in the post-traumatic period.

\section{Conclusion}

The studies have shown that the burn wound tissue shows a higher level of the real part of the dielectric permittivity in comparison with intact skin, and these changes have a temporal dynamics. So, immediately after the burn, the parameter change prevails in the surface layers of biological tissue, and after one day - in the deeper layers.

The stability of this pattern determines the possibility of using the method of microwave profiling of the skin in the assessment of its structure in normal and local changes (benign and malignant tumors, burns, etc.), and the equations can serve as a guide for the subsequent study of the dielectric characteristics of the cover tissues of mature Wistar rats in diverse experiments.

\section{References}

1. Arai T. Burns. Nihon Rinsho. 2016. V. 74, No 2. P. 231-235.

2. Daigeler A, Kapalschinski N, Lehnhardt M. Therapy of burns. Chirurg. 2015. V. 86, No 4. P. 389-401.

3. Ida T., Iwazaki H., Kawaguchi Y. et al. Burn depth assessments by photoacoustic imaging and laser Doppler imaging. Wound Repair Regen. 2016. V. 24, No 2. P. 349355 .

4. Li H., Zhang J., Chen J. et al. Integration of burn treatment and rehabilitation for a child with extremely severe burn. Zhonghua Shao Shang Za Zhi. 2015. V. 31, No 2. P. 130-134.

5. Kostrov A.V., Smirnov A.I., Yanin D.V. et al. Resonanse near-field microwave diagnostics of non-homogenous mediums. Izvestiya RAS. Ser, Phys. 2005. V. 69, No 12. P. 1716-1720.

6. Peretyagin S.P., Martusevich A.K., Vazina I.R. et al. Development of new method of combined thermal trauma modeling. Sovremennye techologgii v meditsine. 2011. No 2. P. 106-109. 